\title{
Magnetoelectric effect in metal-PZT laminates
}

\author{
S NARENDRA BABU*, T BHIMASANKARAM and S V SURYANARAYANA \\ Department of Physics, Osmania University, Hyderabad 500 007, India
}

MS received 20 December 2004

\begin{abstract}
Magnetoelectric (ME) composites are two-phase composites consisting of piezoelectric and piezomagnetic materials as the participating constituents. These magnetoelectric composites when placed under external magnetic field, show electrical polarization (magnetoelectric output). The ME coupling is mediated by mechanical stress. In the present study, we have synthesized $\mathrm{Ni} / \mathrm{PZT} / \mathrm{Ni}$ and $\mathrm{Fe} / \mathrm{PZT} / \mathrm{Fe}$ layered composites for studying their ME output by dynamic magnetoelectric set up in which both d.c. and a.c. magnetic fields can be varied. The ME output obtained in these composites are higher than those obtained in $40 \% \mathrm{Ni}_{0.97} \mathrm{Co}_{0.03} \mathrm{Mn}_{0.01}$ $\mathrm{Fe}_{1.9} \mathrm{O}_{4}+60 \% \mathrm{BaTi}_{1.02} \mathrm{O}_{3.04}$. The results with varying d.c. and a.c. magnetic fields are presented.
\end{abstract}

Keywords. Magnetoelectric effect; composite; laminate; PZT.

\section{Introduction}

The magnetoelectric (ME) effect, the appearance of an electric polarization (ME output) on applying a magnetic field (or) by appearance of magnetization on applying an electric field, is a predominant property observed in two phase composites consisting of piezoelectric and piezomagnetic materials which is absent in either of the phases (Suryanarayana 1994). The deformation of piezomagnetic phase causes polarization of piezoelectric phase in the composite. On the other hand the electrical polarization of piezoelectric material causes change in magnetization of piezomagnetic phase due to the mechanical coupling of the piezomagnetic and piezoelectric phases (Lopatin et al 1994). Magnetoelectric composites are exploited as sensors, waveguides, modulators, switches and phase inverters (Brache and Van Vliet 1981).

In the literature, various composites have been reported. These are $\mathrm{Ni}(\mathrm{Co}, \mathrm{Mn}) \mathrm{Fe}_{2} \mathrm{O}_{4}-\mathrm{BaTiO}_{3}, \mathrm{CoFe}_{2} \mathrm{O}_{4}-\mathrm{BaTiO}_{3}$, $\mathrm{NiFe}_{2} \mathrm{O}_{4}-\mathrm{BaTiO}_{3}, \quad \mathrm{LiFe}_{5} \mathrm{O}_{8}-\mathrm{BaTiO}_{3}, \quad \mathrm{CoFe}{ }_{2} \mathrm{O}-\mathrm{Bi}_{4} \mathrm{Ti}_{3} \mathrm{O}_{12}$, $\mathrm{PZT}-\mathrm{CoFe}_{2} \mathrm{O}_{4}$ NCF-PZT (Vanden Boomgaard et al 1974, 1976; Van Run et al 1974; Lupeiko et al 1994, 1995; Yu et al 1996; Srinivas 2001; Srinivas et al 2002). Srinivas et al (2002) evaluated the electromechanical coupling coefficients in $50 \%$ PZT and $50 \% \quad \mathrm{CoFe}_{2} \mathrm{O}_{4}$ composite.

Srinivas (2001) investigated the microstructures, piezoelectric and $\mathrm{ME}$ properties of $\mathrm{Ni}_{0.98} \mathrm{Co}_{0.02} \mathrm{Fe}_{1.9} \mathrm{Mn}_{0.02} \mathrm{O}_{4}$ (NCF) and PZT matrix and reported a ME voltage of $160 \mathrm{mV} / \mathrm{cm}$ in $60 \% \mathrm{NCF}-40 \%$ PZT composite which was $62 \%$ higher than the previously reported value for the ME particulate composite (Vanden Boomgaard et al 1976).

\footnotetext{
*Author for correspondence (narendra_sim@yahoo.co.in)
}

According to Jungho Ryu and co-workers (2001a), the fabrication technique of the laminate composites (2-0 composite) on a macroscopic scale can have advantages in tailoring design patterns for magnetic noise sensing. They reported a high ME coefficient of $4.68 \mathrm{~V} / \mathrm{cm}-\mathrm{Oe}$ at room temperature for a laminate consisting of Terfenol-DPZT. They also observed ME output dependence on the direction of an a.c. magnetic field under a d.c. bias field and reported a very high $\mathrm{ME}$ coefficient $(\mathrm{d} E / \mathrm{d} H)$ at $1 \mathrm{kHz}$ for the composite as $5 \cdot 90 \mathrm{~V} / \mathrm{cm}-\mathrm{Oe}$ (Jungho Ryu et al 2001b).

Kiyotakemori and Wuttig (2002) reported an $\mathrm{ME}_{\mathrm{H}}$ coefficient of $1.43 \mathrm{~V} / \mathrm{cm}-\mathrm{Oe}$ in the Terfenol-D-PVDF composite. In laminate composites of PMN-PT single crystal and Terfenol-D, Jungho Ryu et al (2002) reported an ME coefficient of $10 \cdot 30 \mathrm{~V} / \mathrm{cm}-\mathrm{Oe}$.

So far the reported work on magnetoelectric composites has been on mostly involving ceramic oxides or a combination of an intermetallic and a ferroelectric oxide. The samples were prepared either as bulk by solid state sintering or as laminates or recently as thin films (Chang et al 2004). Magnetoelectric coefficients, $\alpha_{E}$, of the bulk particulate composites of ferrite-piezoelectric ceramics were 2 or 3 orders of magnitude smaller than theoretical predictions. Such low values are primarily due to low resistivity for ferrites, which (i) limits the electric field used for poling the composite and consequently a poor piezoelectric coupling and (ii) produces a leakage current that results in the loss of induced voltage. However, higher ME output values were reported in layered laminates (Srinivasan et al 2001; Jungho Ryu et al 2002). While studying the frequency dependence of ME interactions in Permendur and PZT laminates, Laletsin et al (2004) reported the maximum ME coefficient of $90 \mathrm{~V} / \mathrm{cm}-\mathrm{Oe}$ at the resonant frequency of PZT.

In this present work, we report the fabrication of laminate composite with a metal (i.e. $\mathrm{Ni}$ and $\mathrm{Fe}$ ) and PZT. 
The magnetostriction value of $\mathrm{Ni}$ varies between $-32 \times$ $10^{-6}$ and $-35 \times 10^{-6}$ while that for Fe it varies from $-8 \times$ $10^{-6}$ to $-10 \times 10^{-6}$. The ME properties of metal-PZT laminates with varying thickness of PZT from 0.1$1 \mathrm{~mm}$, at a constant thickness $(1 \mathrm{~mm})$ of metal are being reported in this paper.

\section{Experimental}

\subsection{Materials}

The magnetic phases chosen were $\mathrm{Ni}$ and Fe. The desired dimensions of $\mathrm{Ni}$ and $\mathrm{Fe}$ specimens were cut from spec pure $(99.9+\%)$ metals obtained from Chempure. The PZT disks were cut from the samples (C-52) obtained from $\mathrm{M} / \mathrm{s}$ Concord Electroceramics Ltd, New Delhi. PZT was chosen as the piezoelectric phase to prepare the laminates, in view of its high piezoelectric constants.

\subsection{Sample preparation}

PZT pellets were obtained with different thicknesses of $1 \mathrm{~mm}, 0.5 \mathrm{~mm}$ and $0.4 \mathrm{~mm}$ with a diameter of $5 \mathrm{~mm}$ and $12 \mathrm{~mm}$. $\mathrm{Ni}$ discs were machined to dimensions of $\Phi 5 \mathrm{~mm} \times 1 \mathrm{~mm}$ and $\mathrm{Fe}$ discs were machined to dimensions of $\Phi 12 \mathrm{~mm} \times 1 \mathrm{~mm}$. Ni/Fe and PZT discs were stacked using conducting epoxy and cured at $80^{\circ} \mathrm{C}$ for $1 \mathrm{~h}$ and $100^{\circ} \mathrm{C}$ for $30 \mathrm{~min}$. The schematic geometry of a laminar composite sample is shown in figure 1 . The di-

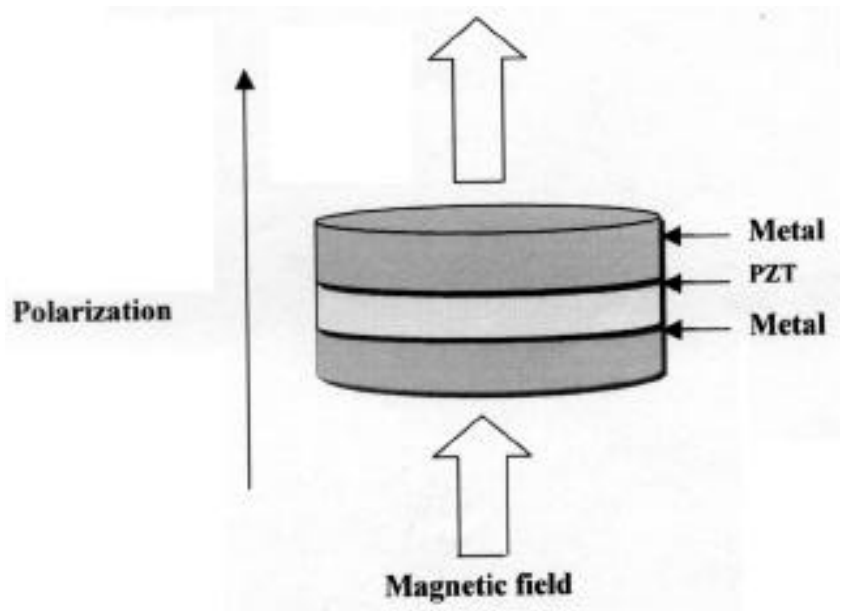

Figure 1. The schematic structure of a laminate composite. mensions of the prepared samples are given in table 1 . PZT pellets were electroded by silver paint and electrically polarized under an electric field of $30 \mathrm{kV} / \mathrm{cm}$ for $10 \mathrm{~min}$.

\section{Measurements}

An indigenous experimental set up (Mahesh Kumar et al 1998) for the measurement of dynamic magnetoelectric effect has been used. The samples are kept between the pole pieces of a d.c. magnet, which can generate the d.c. magnetic field up to $5 \mathrm{kOe}$. The magnetoelectric voltage coefficient is determined by measuring the electric field generated across the sample when an a.c. magnetic field and a d.c. bias are applied to it.

In the dynamic method, the ME output is measured at a constant a.c. magnetic field of $64 \mathrm{Oe}(f=1.008 \mathrm{kHz})$ superimposed on a varying d.c. field in the range of 1-4.5 kOe. $\mathrm{ME}$ conversion in these laminate composites were recorded as a function of d.c. bias field at an a.c. field of $64 \mathrm{Oe}(f=1.008 \mathrm{kHz})$.

The ME measurements have also been carried out as function of varying a.c. field. A varying a.c. magnetic field $(f=1.008 \mathrm{kHz})$ is superimposed over constant d.c. magnetic fields of $1 \mathrm{kOe}$ to $4.5 \mathrm{kOe}$ in steps of $0.5 \mathrm{kOe}$ for measuring magnetoelectric output.

\section{Results and discussion}

In the dynamic method the ME output is observed at a fixed a.c. magnetic field range of 2 Oe to $64 \mathrm{Oe}$. At all these a.c. magnetic fields, the ME output is constant with varying d.c. magnetic field. Figure 2 depicts the variation of magnetoelectric (ME) output with d.c. magnetic field bias. The ME output of sample $S_{1}$ (please refer to table 1 for the nomenclature) is higher than that of samples $S_{2}$ and $S_{3}$. The ME output of $\mathrm{Ni} / \mathrm{PZT} / \mathrm{Ni}$ samples is almost constant with the d.c. magnetic field in the range $1 \mathrm{kOe}-$ $4.5 \mathrm{kOe}$.

The maximum ME output obtained for sample $S_{1}$ is $169.5 \mathrm{mV} / \mathrm{cm}$ at a fixed 64 Oe a.c. magnetic field $(1.008 \mathrm{kHz}$ frequency) and $1 \mathrm{kOe}$ d.c. magnetic field (table 2). The value of $\mathrm{ME}$ output is higher than that reported in literature for $40 \% \mathrm{Ni}_{0.97} \mathrm{Co}_{0.03} \mathrm{Mn}_{0.01} \mathrm{Fe}_{1.9} \mathrm{O}_{4}+60 \% \mathrm{BaTi}_{1 \cdot 02} \mathrm{O}_{3.04}$ (Brache and Van Vliet 1981). The ME output values of

Table 1. Dimensions of Ni/PZT/Ni and Fe/PZT/Fe layered composites.

\begin{tabular}{lccrcc}
\hline Metal & Sample & $t_{\text {metal }}$ & $t_{\text {PZT }}$ & $t_{\text {total }}$ & $t_{\text {metal }} / t_{\text {PZT }}$ \\
\hline \multirow{3}{*}{$\mathrm{Ni}$} & $S_{1}$ & $1 \mathrm{~mm}$ & $1 \mathrm{~mm}$ & $3 \mathrm{~mm}$ & 1 \\
& $S_{2}$ & $1 \mathrm{~mm}$ & $0.5 \mathrm{~mm}$ & $2.5 \mathrm{~mm}$ & 2 \\
\multirow{2}{*}{$\mathrm{Fe}$} & $S_{3}$ & $1 \mathrm{~mm}$ & $0.4 \mathrm{~mm}$ & $2.4 \mathrm{~mm}$ & 2.5 \\
& $S_{4}$ & $1 \mathrm{~mm}$ & $1 \mathrm{~mm}$ & $3 \mathrm{~mm}$ & 1 \\
\hline
\end{tabular}


samples $S_{2}$ and $S_{3}$ are even though smaller than sample $S_{1}$ they are higher than the ME output value reported for ferrite-piezoelectric composite (Srinivas et al 2002).

Figure 2 also depicts the variation of magnetoelectric (ME) output with d.c. magnetic field bias for the samples $S_{1}(\mathrm{Ni} / \mathrm{PZT} / \mathrm{Ni})$ and $S_{4}(\mathrm{Fe} / \mathrm{PZT} / \mathrm{Fe})$. The ME output of $\mathrm{Ni} / \mathrm{PZT} / \mathrm{Ni}$ is higher than $\mathrm{Fe} / \mathrm{PZT} / \mathrm{Fe}$ (table 2). The maximum ME output value obtained for $\mathrm{Ni} / \mathrm{PZT} / \mathrm{Ni}$ sample is $169.5 \mathrm{mV} / \mathrm{cm}$ at a fixed $64 \mathrm{Oe}$ a.c. magnetic field $(1.008 \mathrm{kHz}$ frequency) and $1 \mathrm{kOe}$ d.c. magnetic field, whereas $91.1 \mathrm{mV} / \mathrm{cm}$ was obtained at a fixed 64 Oe a.c. magnetic field (1.008 kHz frequency) and $1 \mathrm{kOe}$ d.c. magnetic field for the sample $S_{4}(\mathrm{Fe}-\mathrm{PZT}-\mathrm{Fe})$. The $\mathrm{ME}$ output of $\mathrm{Ni}-$ $\mathrm{PZT}-\mathrm{Ni}$ laminate is evidently higher than $\mathrm{Fe}-\mathrm{PZT}-\mathrm{Fe}$ laminate, as the magnetostriction of $\mathrm{Ni}$ is greater than that of Fe.

The ME voltage coefficient $(\alpha)$ is calculated using the formula (Jungho Ryu et al 2001a)

$$
\alpha=\frac{1}{t}\left(\frac{\delta V}{\delta H}\right)
$$

where, $\delta H$ is an a.c. magnetic field applied to a biased

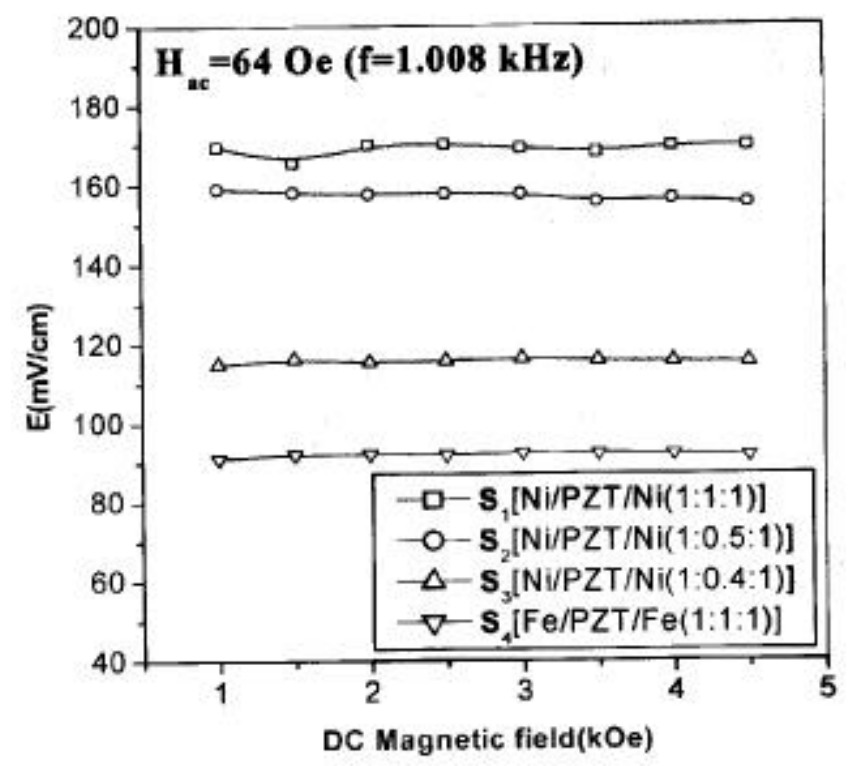

Figure 2. D.C. magnetic field vs magnetoelectric output of Ni-PZT-Ni and Fe-PZT-Fe samples. composite, $\delta V$ the induced voltage and $t$ the thickness of the piezoelectric phase.

The variation of $M E$ coefficient, $\alpha_{\max }$, with d.c. magnetic field for the samples of $\mathrm{Ni} / \mathrm{PZT} / \mathrm{Ni}$ with different thicknesses of PZT and Fe/PZT/Fe is shown in figure 3. The ME coefficient of sample $S_{1}$ is higher than the ME coefficients of samples $S_{2}, S_{3}$ and $S_{4}$. The maximum magnetoelectric coefficient $(\mathrm{d} E / \mathrm{d} H)_{\max }=8.5 \mathrm{mV} / \mathrm{cm}-\mathrm{Oe}$ is obtained for $S_{1}$ sample at fixed 3 Oe a.c. magnetic field $(1.008 \mathrm{kHz}$ frequency) and $1 \mathrm{kOe}$ d.c. magnetic field. Magnetoelectric coefficient $(\mathrm{d} E / \mathrm{d} H)_{\max }=4.5 \mathrm{mV} / \mathrm{cm}-\mathrm{Oe}$ is obtained at fixed 3 Oe a.c. magnetic field $(1.008 \mathrm{kHz}$ frequency) and $1 \mathrm{kOe}$ d.c. magnetic field for the sample $S_{4}$ (Fe-PZT-Fe). The magnetoelectric coefficients of samples $S_{2}$ and $S_{3}$ vary between 5 and $8 \mathrm{mV} / \mathrm{cm}-O e$.

Figure 4 depicts the variation of magnetoelectric (ME) output with a.c. magnetic field $(1.008 \mathrm{kHz})$ at fixed d.c. magnetic bias for $\mathrm{Ni} / \mathrm{PZT} / \mathrm{Ni}$ and $\mathrm{Fe} / \mathrm{PZT} / \mathrm{Fe}$ samples. In this measurement the d.c. magnetic field is fixed in the range $1-4.5 \mathrm{kOe}$ in steps of $0.5 \mathrm{kOe}$ d.c. magnetic field. Magnetoelectric output values of the Ni/PZT/Ni samples with variation of a.c. magnetic field at fixed $1 \mathrm{kOe}$ d.c. magnetic field are shown in figure 4. The ME output

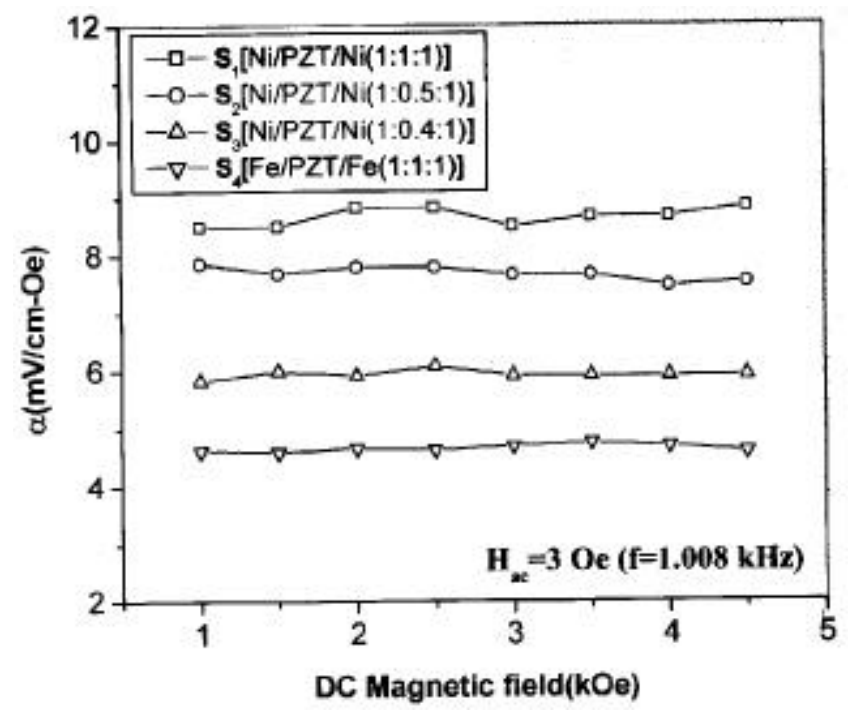

Figure 3. D.C. magnetic field vs magnetoelectric coefficient of Ni-PZT-Ni and Fe-PZT-Fe samples.

Table 2. Magnetoelectric properties of Ni/PZT/Ni and Fe/PZT/Fe layered composites.

\begin{tabular}{ccccccc}
\hline & & \multicolumn{2}{c}{$\begin{array}{c}\text { ME output, } \\
E(\mathrm{mV} / \mathrm{cm})\end{array}$} & & \multicolumn{2}{c}{$\begin{array}{c}\text { ME coefficient, } \\
\alpha(\mathrm{mV} / \mathrm{cm}-\mathrm{Oe})\end{array}$} \\
\cline { 3 - 4 } \cline { 6 - 7 } Metal & Sample & at 3 Oe & at 64 Oe & & at 3 Oe & at 64 Oe \\
\hline \multirow{2}{*}{$\mathrm{Ni}$} & $S_{1}$ & 25.5 & 169.5 & & 8.5 & 2.6 \\
& $S_{2}$ & 23.4 & 159 & & 7.8 & 2.4 \\
$\mathrm{Fe}$ & $S_{3}$ & 17.4 & 115 & & 5.8 & 1.7 \\
& $S_{4}$ & 13.8 & 91.1 & & 4.6 & 1.4 \\
\hline
\end{tabular}




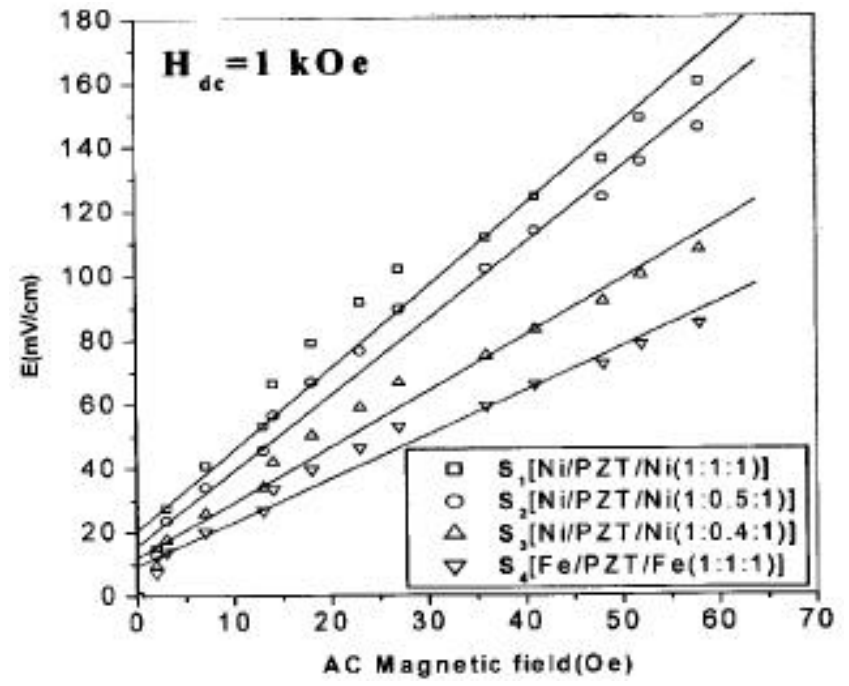

Figure 4. A.C. magnetic field vs magnetoelectric output of $\mathrm{Ni}-\mathrm{PZT}-\mathrm{Ni}$ and $\mathrm{Fe}-\mathrm{PZT}-\mathrm{Fe}$ samples.

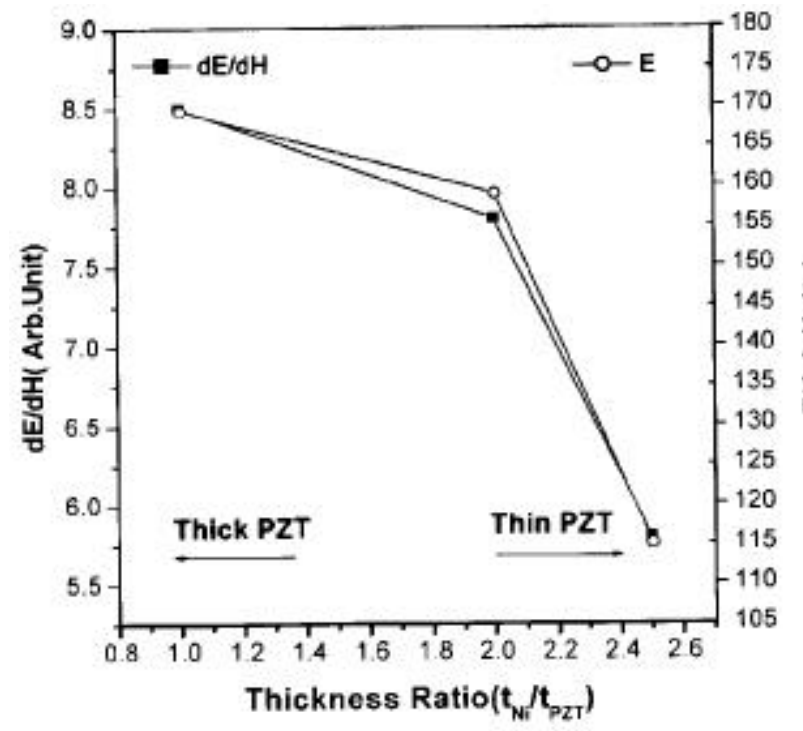

Figure 5. Thickness ratio vs $\mathrm{d} E / \mathrm{d} H \& E$ of Ni-PZT-Ni samples.

linearly increases with increase in a.c. magnetic field for all the four samples. Among $S_{1}, S_{2}, S_{3}$ and $S_{4}$ samples, $S_{1}$ sample exhibits higher ME output. The slope of the plot (magnetoelectric coefficient, $\Delta E / \Delta H$ ) is found to be $2.48 \mathrm{mV} / \mathrm{cm}$-Oe for sample $S_{1}$.

The variation of ME output and magnetoelectric coefficient with thickness ratio for the samples of $\mathrm{Ni} / \mathrm{PZT} / \mathrm{Ni}$ with different thicknesses of PZT, is shown in figure 5. It is observed that with increasing thickness ratio, both the
ME output and magnetoelectric coefficient decrease. Finally, it is concluded that the ME output can be realized in the laminates of metal/ferroelectric composites. Thus a systematic search of combination of various metallic materials, intermetallics and metglasses with piezoelectrics may result in a better combination of a composite, which would show higher ME output for applications.

\section{Acknowledgements}

The authors are grateful to the Aeronautical Development Agency, Bangalore, for providing financial support to carry out this research work. One of the authors (SNB) thanks Dr K Srinivas, ASL, Hyderabad, for useful discussions.

\section{References}

Bokhan Yu I and Latetin V M 1996 Inorg. Mater. 32634

Bracke L P M and Van Vliet R G 1981 Int. J. Electron. 5255

Chang K S et al 2004 Appl. Phys. Letts 843091

Jungho Ryu, Alfredo Vazquez Carazo, Kenji Uchino and HyounEe Kim 2001a Jpn. J. Appl. Phys. 404948

Jungho Ryu, Shashank Priya, Alfredo Vazquez Carazo, Kenji Uchino and Hyoun-Ee Kim 2001b J. Am. Ceram. Soc. 842905

Jungho Ryu, Shashank Priya, Kenji Uchino, Hyoun-Ee Kim and Dwight Viehland 2002 J. Korean Ceram. Soc. 39813

Laletsin U, Paddubnaya N, Srinivasan G and De Vreugd C P 2004 Appl. Phys. A78 33

Lopatin S, Lopatine I and Lisnevskaya I 1994 Ferroelectrics 16263

Lupeiko T G, Lopatin S S, Lisnevskaya I V and Zvyaginstev B I 1994 Inorg. Mater. 301353

Lupeiko T G, Lisnevskaya I V, Chkheidze M D and Zvyaginstev B I 1995 Inorg. Mater. 311139

Mahesh Kumar M, Srinivas A, Suryanarayana S V, Kumar G S and Bhimasankaram T 1998 Bull. Mater. Sci. 21251

Mori K and Wuttig M 2002 Appl. Phys. Letts 81100

Srinivasan G, Rasmussen E T, Gallegos J, Srinivasan R, Bokhan Yu I and Laletin V M 2001 Phys. Rev. B65 214408

Srinivas K 2001 Synthesis and characterization of NCF: PZT magnetoelectric composites, Ph D Thesis, Osmania University, Hyderabad

Srinivas K, Prasad G, Bhimasankaram T and Suryanarayana S V 2002 Mod. Phys. Letts B14 663

Suryanarayana S V 1994 Bull. Mater. Sci. 171259

Vanden Boomgaard J, Terrell D R, Born R A J and Giller H F J I 1974 J. Mater. Sci. 91705

Vanden Boomgaard J, Van Run A M J G and Van Suchtelen J 1976 Ferroelectrics 10295

Van Run A M J G, Terrell D R and Scholing J H 1974 J. Mater. Sci. 91710 